Please do not remove this page

RMIT

UNIVERSITY

\title{
Multipath mobile channel model for microcell environment
}

Sharif Mahmoud, Seedahmed; Hussain, Zahir; O'Shea, Peter

https://researchrepository.rmit.edu.au/esploro/outputs/9921858175201341/filesAndLinks?institution=61RMIT_INST\&index=null

Sharif Mahmoud, S., Hussain, Z., \& O'Shea, P. (2004). Multipath mobile channel model for microcell environment. Proceedings of the 2004 IEEE International Symposium on Spread Spectrum Techniques and Applications, 87-91.

https://researchrepository.rmit.edu.au/discovery/fulldisplay/alma9921858175201341/61RMIT_INST:Resea rchRepository

Repository homepage: https://researchrepository.rmit.edu.au

(c) 2004 IEEE. Personal use of this material is permitted. However, permission to reprint/republish this material for advertising or promotional purposes or for creating new collective works for resale or redistribution to servers or lists, or to reuse any copyrighted component of this work in other works must be obtained from the IEEE.

Downloaded On 2023/04/27 00:30:09 +1000 


\title{
A Multipath Mobile Channel Model for Microcell Environment
}

\author{
Seedahmed S. Mahmoud*, Zahir M. Hussain*, and Peter O'Shea ${ }^{\dagger}$ \\ ${ }^{*}$ School of Electrical and Computer Engineering \\ RMIT University, Melbourne, Victoria 3000, Australia \\ E-mail: s2113794@student.rmit.edu.au,zahir.hussain@rmit.edu.au \\ ${ }^{\dagger}$ School of Electrical and Electronic Systems Engineering \\ Queensland University of Technology, Brisbane, Queensland 4000, Australia \\ E-mail: pj.oshea@qut.edu.au
}

\begin{abstract}
We present a geometric multipath propagation model for a microcell mobile environment. The model provides the statistics for the direction-of-arrival (DOA) of multipath components; also it provides the multipath power delay profiles (PDP), which are used to evaluate the bit error rate (BER) and the signal to interference ratio (SIR) for the direct-sequence code division multiple access (DS-CDMA). The probability density function (pdf) for the DOA under the proposed model is compared with experimental results for indoor multipath channel. The experimental results agreed with the proposed model's simulation results.
\end{abstract}

\section{INTRODUCTION}

Channel modelling is an important issue for design and analysis of mobile communication systems. A signal propagating through a wireless channel usually arrives at the destination along a number of different paths, referred to as multipaths [1]. The received signal is much weaker than the transmitted signal due to phenomena such as mean propagation loss, slow fading, and fast fading [1]. For analysing the performance of wireless communication systems, a statistical channel model (which provides information about the DOA and time-ofarrival (TOA) of the multipath components) is required.

In this paper we present a statistical channel model for a microcell environment. This model assumes that there is a lineof-sight (LOS) path between the transmitter and the receiver, and that the scatterers are arranged in a circle of radius $R$ centered on the mobile station (MS). It is further assumed that the base station (BS) lies within this circle. The distances $r_{k}$ between the scatterers and the MS conform to a hyperbolic distribution. This assumption is more realistic and flexible than other commonly used probability density functions (pdf's), such as the uniform pdf [1]. This is so because the hyperbolic distribution allows scatterers to assume higher concentration in a flexible area in the vicinity of the mobile. Validation of this claim using practical data will be presented in Section III. The actual spread is decided by a practical parameter that depends on the specific physical environment. For example, in an urban area the mobile would see a large number of scatterers in the immediate neighborhood, while farther buildings can also act as scatterers for waves that escaped the immediate neighborhood, but the farther we go from the mobile the fewer are the buildings that may act as scatterers, as they will be obscured by nearer buildings. The proposed model assumes that each multipath component is created by a specular reflection of the propagating signal at the remote object. Once the co-ordinates of the scatterers are drawn from the reciprocal cosh distribution of $r_{k}$, all necessary channel characteristics, including DOA, can be derived [2], [3].

The joint pdf of the power-DOA and the power-Doppler frequency shift for the proposed channel model are presented and simulated. Further, we study the performance of the DSCDMA system over the proposed channel model. The multipath PDP arising from this channel for different propagation scenarios have been used as inputs to the simplified expression for the improved Gaussian approximation (SEIGA) approach to evaluate the BER and the SIR for the DS-CDMA.

\section{The Channel Model}

In this section we introduce a geometrically based channel model with hyperbolically distributed scatterers for LOS microcell environment. Signals received at the base station are assumed to be plane waves arriving from the horizon and hence the DOA calculation will include only the azimuthal coordinate [1].

Fig.1 illustrates the proposed channel model. This model assumes that the scatterers are arranged within a circle of radius $R$ around the mobile. The BS is within the scatterers' circle. The distances $r_{k}$ between the mobile and the scatterers are distributed according to an inverse-cosh-squared distribution, whereas the angles of departure, $\psi_{k}$, are uniformly distributed in the interval, $[0,2 \pi] . D$ denotes the distance between the BS and the MS. This model is suitable for a microcell environment where $D<R$, and the BS, MS and scatterers are all in relatively close proximity. The angle $\theta_{k}$ is the DOA for the $\mathrm{k}^{\text {th }}$ path delay.

The baseband complex envelope assuming omnidirectional antennas, a stationary user, and a multipath channel impulse response $h_{b}$ is given by [1]

$$
h_{b}(t, \tau)=\sum_{k=0}^{L-1} \alpha_{k} \delta\left(t-\tau_{k}\right)=\sum_{k=0}^{L-1} \beta_{k} e^{j \phi_{k}} \delta\left(t-\tau_{k}\right)
$$




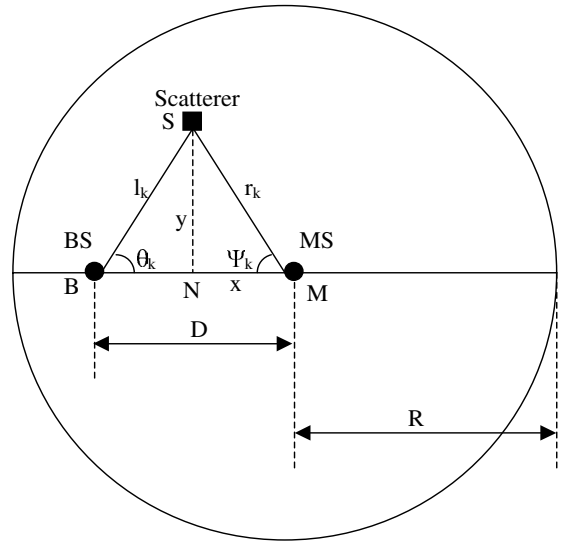

Fig. 1. Geometry of the Proposed Model. "MS" stands for the mobile station, and "BS" is the base station

where $\beta_{k}=\left|\alpha_{k}\right|$ is the magnitude of the $\mathrm{k}^{\text {th }}$ multipath component, $\phi_{k}$ is the phase of the multipath component, $\tau_{k}$ is the path delay, and $L$ is the number of multipaths. We assume that a LOS path exists with time-of-arrival given by $\tau_{0}=\frac{D}{c}$, where $c$ is the speed of light. (1) would need to be modified if omni-directional antennas are not used at both the MS and the BS. To model the system performance with multiple antennas, it is necessary to model the DOA [1].

\section{A. The Proposed Model Parameters}

The probability density functions of $r_{k}$ and $\psi_{k}$ are proposed to be as follows

$$
\begin{gathered}
f_{r_{k}}\left(r_{k}\right)=\frac{a}{\tanh (a R) \cosh ^{2}\left(a r_{k}\right)} \text { where } 0 \leq r_{k} \leq R \\
f_{\psi_{k}}\left(\psi_{k}\right)=\frac{1}{2 \pi} \quad \text { where } \quad 0 \leq \psi_{k} \leq 2 \pi
\end{gathered}
$$

where the scattering spread parameter $a$ can assume values in the interval $(0,1)$. A suitable value of $a$ must be chosen for the simulation. Conveniently, formula (10) in [4] relates $a$ to the angle spread. Thus, for a given angle spread (assumed or measured), one can used the formula in [4] to deduce an appropriate value of $a$. The radius of the scatterers' circle, $R$, controls by the scattering spread parameter $a$, while the distance $D$ between the MS and the BS is chosen to be equal or within the range of the measurement distance. The cumulative probability functions for (2) and (3) are given by

$$
\begin{gathered}
F_{r_{k}}\left(r_{k}\right)=\left\{\begin{array}{lc}
\frac{\tanh \left(a r_{k}\right)}{\tanh (a R)} & \text { for } 0 \leq r_{k} \leq R \\
0 & \text { elsewhere }
\end{array}\right. \\
F_{\psi}\left(\psi_{k}\right)=\frac{\psi_{k}}{2 \pi} \quad \text { for } 0 \leq \psi_{k} \leq 2 \pi
\end{gathered}
$$

For simulation purposes, generation of samples for the random variables $r_{k}$ and $\psi_{k}$ can be done by using a common random number generator followed by application of some appropriate transformations. The common random number generator draws samples $\mathrm{x}_{k}$ and $\mathrm{y}_{k}$ from uniformly distributed random variables $\mathrm{X}$ and $\mathrm{Y}$ in the interval $[0,1]$. These samples are then transformed to create $r_{k}\left(x_{k}\right)$ and $\psi_{k}\left(y_{k}\right)$ using transformations derived from (4) and (5). These transformations are

$$
\begin{gathered}
r_{k}=\frac{1}{a} \tanh ^{-1}\left(x_{k} \tanh (a R)\right) \\
\psi_{k}=2 \pi y_{k} .
\end{gathered}
$$

The path delay $\tau_{k}$ of the $\mathrm{k}^{\text {th }}$ multipath component is given by

$$
\tau_{k}=\frac{\left(l_{k}+r_{k}\right)}{c}=\frac{1}{c}\left(r_{k}+\sqrt{r_{k}^{2}+D^{2}-2 r_{k} D \cos \left(\psi_{k}\right)}\right) .
$$

The DOA for the $\mathrm{k}^{\text {th }}$ path, $\theta_{k}$, is given over $(-\pi, \pi)$ by

$$
\theta_{k}=\left\{\begin{array}{l}
\tan ^{-1}\left(\frac{r_{k} \sin \left(\psi_{k}\right)}{D-r_{k} \cos \left(\psi_{k}\right)}\right) \\
\operatorname{for} r_{k} \cos \left(\psi_{k}\right) \leq D ; \text { or } \\
\tan ^{-1}\left(\frac{r_{k} \sin \left(\psi_{k}\right)}{D-r_{k} \cos \left(\psi_{k}\right)}\right)+\pi \\
\operatorname{for}\left(r_{k} \cos \left(\psi_{k}\right)>D\right) \&\left(0 \leq \psi_{k} \leq \frac{\pi}{2}\right) ; \text { or } \\
\tan ^{-1}\left(\frac{r_{k} \sin \left(\psi_{k}\right)}{D-r_{k} \cos \left(\psi_{k}\right)}\right)-\pi \\
\text { for }\left(r_{k} \cos \left(\psi_{k}\right)>D\right) \&\left(\frac{3 \pi}{2} \leq \psi_{k} \leq 2 \pi\right)
\end{array}\right.
$$

The mean power of each multipath component depends on the propagation delay $\tau_{k}$, and is usually defined by a characteristic $\mathrm{PDP}, \mathrm{P}\left(\tau_{k}\right)$, which is given by [1]

$$
P\left(\tau_{k}\right)=P_{\text {ref }}-10 n \log \left(\tau_{k} / \tau_{\text {ref }}\right)
$$

In the above $n$ is the path loss exponent and it depends on the propagation scenario to be simulated. $P_{r e f}$ is a reference power that is measured at a distance, $d_{\text {ref }}$, from the transmitting antenna when omnidirectional antennas are used at both the transmitter and the receiver.

The power in each multipath component $P_{k}$ is related to the magnitude $\alpha_{k}$ of the $\mathrm{k}^{t h}$ multipath in equation (1) by

$$
P\left(\tau_{k}\right)=P_{0}+20 \log \left|\alpha_{k}\right| \text {. }
$$

The above equation can be re-arranged to give

$$
\alpha_{k}=10^{\frac{P_{k}-P_{0}}{20}}
$$

where $P_{0}$ is the direct path power in $\mathrm{dB}$.

\section{B. Statistics for the Power-DOA and the Power-Doppler Shift}

In this subsection we will provide the joint power-DOA and the joint power-Doppler shift pdfs for the proposed model. From (2) and Fig. 1, the joint power-DOA $f_{p_{k}, \theta_{k}}\left(p_{k}, \theta_{k}\right)$ for the proposed channel model is found to be as follows

$$
f_{p, \theta}(p, \theta)=\frac{a D\left(D^{2}-D^{2}\left(\frac{p}{p_{o}}\right)^{-\frac{2}{n}}\right) h(p, \theta)}{4 n p_{o} \tanh (a R) \cosh ^{2}(\Phi(p, \theta))}
$$


where, $h(p, \theta)=\frac{\left(D^{2}+D^{2}\left(\frac{p}{p_{o}}\right)^{-\frac{2}{n}}-2 D^{2}\left(\frac{p}{p_{o}}\right)^{-\frac{1}{n}} \cos (\theta)\right)}{\left(\frac{p}{p_{o}}\right)^{\frac{n+1}{n}}\left(D \cos (\theta)-D\left(\frac{p}{p_{o}}\right)^{-\frac{1}{n}}\right)^{3}}$ and $\Phi(p, \theta)=a \sqrt{\frac{(m(p, \theta))^{2}}{4}-D \cos (\theta) m(p, \theta)+D^{2}}$, where $m(p, \theta)=\frac{\left(D^{2}-D^{2}\left(\frac{p}{p_{o}}\right)^{-\frac{2}{n}}\right)}{\left(D \cos (\theta)-D\left(\frac{p}{p_{o}}\right)^{-\frac{1}{n}}\right)}$.

Similarly the joint power-Doppler shift pdf is found to be

$$
f_{p, f_{d}}\left(p, f_{d}\right)=\sum_{i=1}^{2} \frac{a D^{2}\left(1-\xi^{2}\right) \Upsilon\left(\xi, f_{d}\right)}{4 n p_{o} \tanh (a R) \cosh ^{2}\left(\beta\left(\xi, \theta_{i}\right)\right)}
$$

where $\xi=\left(\frac{p}{p_{o}}\right)^{-\frac{1}{n}}$,

$$
\Upsilon\left(\xi, f_{d}\right)=\frac{\left(1+\xi^{2}-2 \xi \cos \left(\theta_{i}\right)\right)}{\xi^{-(n+1)}\left(\cos \left(\theta_{i}\right)-\xi\right)^{3} f_{m} \sqrt{1-\left(\frac{f_{d}}{f_{m}}\right)^{2}}}
$$

and $\beta\left(\xi, \theta_{i}\right)=a \sqrt{\frac{\left(m\left(\xi, \theta_{i}\right)\right)^{2}}{4}-D \cos \left(\theta_{i}\right) m\left(\xi, \theta_{i}\right)+D^{2}}$ where $m\left(\xi, \theta_{i}\right)=\frac{\left(D^{2}-D^{2} \xi^{2}\right)}{\left(D \cos \left(\theta_{i}\right)-D \xi\right)}$, and

$$
\theta_{i}= \begin{cases}\theta_{v}+\cos ^{-1}\left(\frac{f_{d}}{f_{m}}\right) & : i=1 \\ \theta_{v}-\cos ^{-1}\left(\frac{f_{d}}{f_{m}}\right) & : i=2 .\end{cases}
$$

Fig. 2 shows the joint power-DOA pdf $f_{p_{k}, \theta_{k}}\left(p_{k}, \theta_{k}\right)$ (eq. (13)) for the proposed microcell channel model. In this simulation we considered a path loss exponent of 2 (free space), the distance between the mobile and the base station is set to $D=1 \mathrm{~km}$, the scatterers' circle radius is $R=2 \mathrm{~km}$, and the power of the direct line-of-sight path, $p_{o}$, has been limited to 1 Watts.

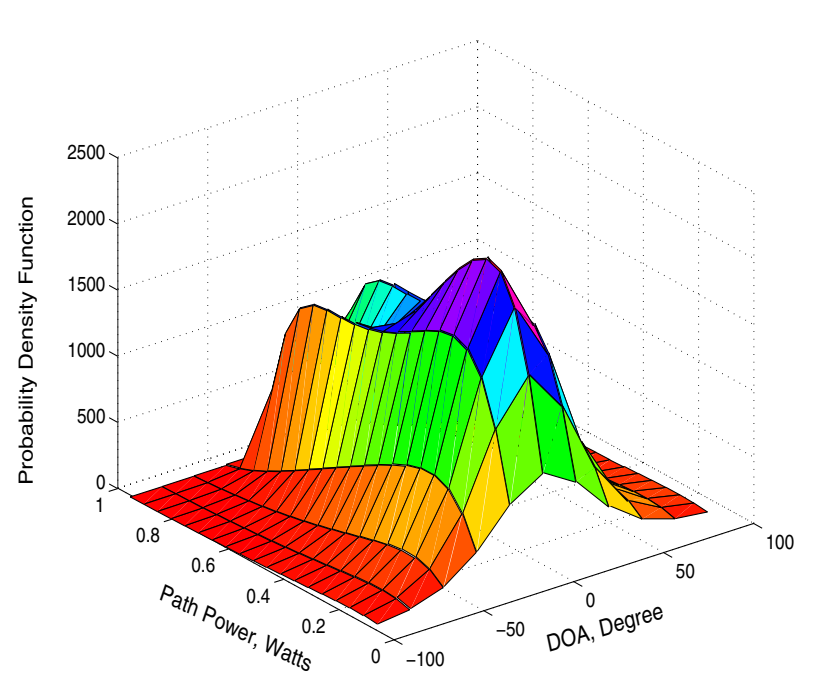

Fig. 2. The Joint power-DOA probability density function for the proposed channel model: $(D=1 \mathrm{~km}, R=2 \mathrm{~km}, a=0.0025, n=2$, and $\left.p_{o}=1 \mathrm{~W}\right)$.

Fig. 3 shows the joint power-Doppler shift pdf for the proposed microcell channel model. In this figure the direction where the mobile is travelling is set to $\theta_{v}=\frac{\pi}{2}$. The parameter $a$ is set to $0.0033, D$ is set to $1 \mathrm{~km}$ and $R=1.5 \mathrm{~km}$.

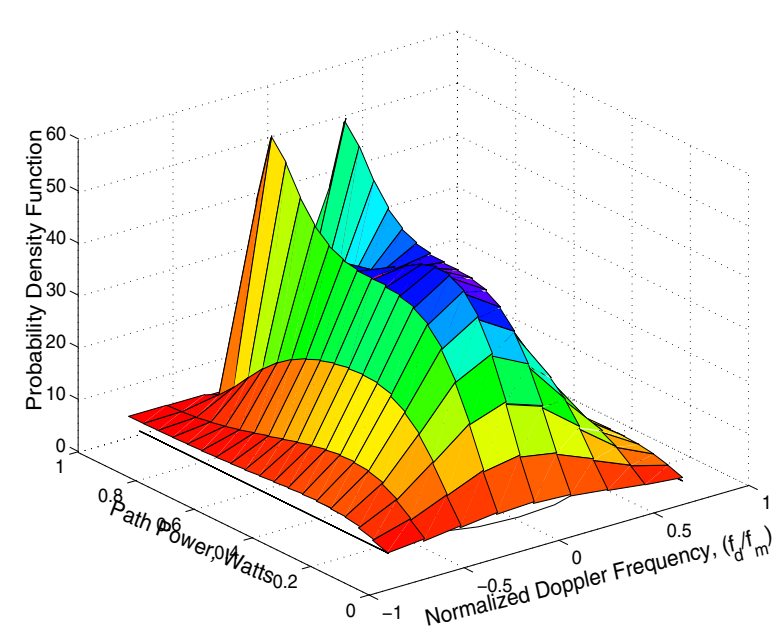

Fig. 3. The Joint power- Doppler frequency probability density function for the proposed channel model: $\left(\theta=\frac{\pi}{2}, D=1 \mathrm{~km}, R=\right.$ $1.5 \mathrm{~km}, a=0.0033, n=2$, and $\left.p_{o}=1 \mathrm{~W}\right)$.

\section{EXPERIMENTAL RESUlTS}

In this section we verify the validity of the proposed model. The DOA pdf for this model is compared with the measurement data reported in [5] and analyzed by Janaswamy [6]. To determine the joint pdf $f(l, \theta)$ for the proposed model, a transformation of the random variable $(x, y)$ into the random variable $(l, \theta)$ is performed by

$$
f_{l, \theta}(l, \theta)=\left.|J(x, y)| f_{x, y}(x, y)\right|_{x=p(l, \theta), y=g(l, \theta)}
$$

where $J(x, y)$ is the Jacobian of the transformation and we restrict it to be positive. From Fig. 1 we get

$$
\begin{gathered}
r=\sqrt{x^{2}+y^{2}} \leq R \\
x=p(l, \theta)=D-l \cos (\theta) \\
y=g(l, \theta)=l \sin (\theta)
\end{gathered}
$$

The Jacobian $J(l, \theta)$ is given by

$$
J(l, \theta)=\left|\begin{array}{ll}
\frac{\partial p}{\partial l} & \frac{\partial p}{\partial \theta} \\
\frac{\partial g}{\partial l} & \frac{\partial g}{\partial \theta}
\end{array}\right|=\left|\begin{array}{rr}
-\cos (\theta) & l \sin (\theta) \\
\sin (\theta) & l \cos (\theta)
\end{array}\right|=-l
$$

By substituting (2), (18), (19), (20) and (21) into (17) we get

$$
f(l, \theta)=\left\{\begin{array}{rr}
\frac{a l}{\tanh (a R) \cosh ^{2}(a h(l, \theta))}, & 0 \leq l \leq(D+R) \\
0 \quad & \text { elsewhere }
\end{array}\right.
$$

where

$$
h(l, \theta)=\sqrt{l^{2}-2 l D \cos (\theta)+D^{2}} .
$$


In [5], Spencer et al. conducted a number of indoor measurement results, collected at $7 \mathrm{GHz}$ within office buildings on the BYU campus. The scanning was done mechanically with a $6^{\circ}$ horn over a $360^{\circ}$ range. At the Clyde building, the angular data measured is for data within one cluster about its mean angle. The angular spread (standard deviations) is $24.5^{\circ}$. Fig. 4 shows a comparison of the results for the DOA pdf for the proposed channel model versus the measurement data reported in [5]. The DOA pdf for the proposed model is evaluated numerically using eq.(22). For the proposed model, the values of $D$ and $a$ were determined to be $100 \mathrm{~m}$ and 0.028 , respectively, to produce the same value of the measurement standard deviations $\left(24.5^{\circ}\right)$.

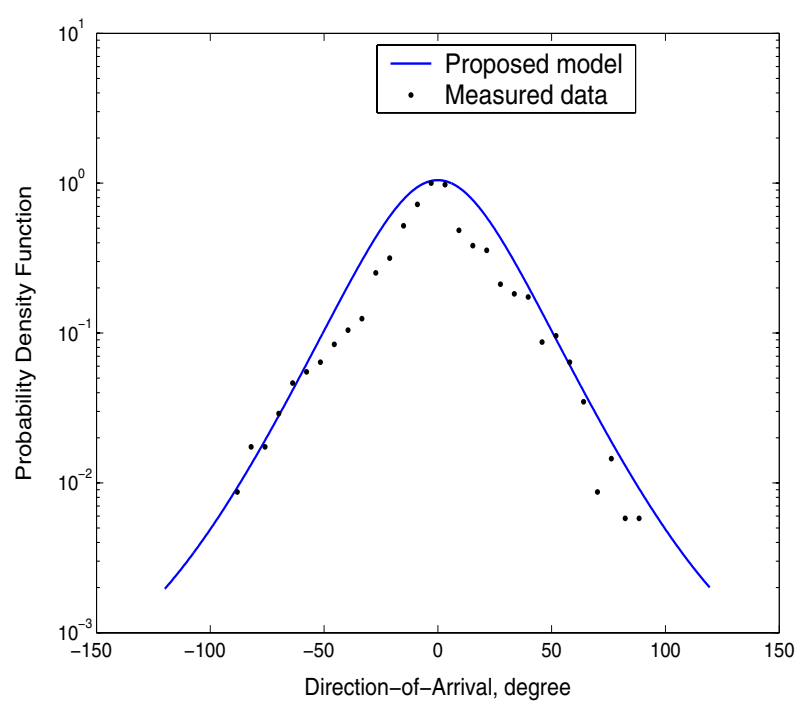

Fig. 4. A comparison of the direction-of-arrival density function for the proposed channel model with the practical measurements described in [5].

From Fig. 4, it is clear that there is a good match between the proposed channel model result and the measurement data, except for a short range of negative angles near the LOS. This is because of the measured data are asymmetric in angle implying that the scattering centers are asymmetric about the LOS direction [5], [6]. This figure shows the validity of the proposed model.

\section{Performance Analysis of DS-CDMA in the PRoposed CHANNEL MOdEL}

We consider the reverse link (mobiles to base station) of a single cell asynchronous DS-CDMA system that supports M active users.

\section{A. BER Performance and SIR Performance}

The SEIGA approach was developed in [7] to estimate the BER in DS-CDMA systems. For a wideband channel model with a line-of-sight, the SEIGA for the bit error probability is given by [8]

$$
\begin{aligned}
\bar{P}_{e} \approx & \frac{2}{3} Q\left(\sqrt{\frac{P_{0}\left|\alpha_{0,0}\right|^{2}}{\mu_{\psi}+\frac{N_{0}}{2 T_{b}}}}\right)+\frac{1}{6} Q\left(\sqrt{\frac{P_{0}\left|\alpha_{0,0}\right|^{2}}{\mu_{\psi}+\sqrt{3} \sigma_{\psi}+\frac{N_{0}}{2 T_{b}}}}\right) \\
& +\frac{1}{6} Q\left(\sqrt{\frac{P_{0}\left|\alpha_{0,0}\right|^{2}}{\mu_{\psi}-\sqrt{3} \sigma_{\psi}+\frac{N_{0}}{2 T_{b}}}}\right)
\end{aligned}
$$

where $\mu_{\psi}$ and $\sigma_{\psi}$ are the mean and standard deviation of $\psi$, respectively, $\psi$ is the variance of the signal-plus-interference portion of the decision statistic, and $Q(n)$ is given by $Q(n)=$ $\frac{1}{\sqrt{2 \pi}} \int_{n}^{\infty} e^{\frac{-u^{2}}{2}} d u$. The mean and standard deviation of $\psi$ are given by the formulas (26), (27) in [8], respectively.

We obtained the following formula for SIR

$$
\begin{aligned}
S I R & \approx \frac{2}{3}\left(\frac{P_{0}\left|\alpha_{0,0}\right|^{2}}{\mu_{\psi}+\frac{N_{0}}{2 T_{b}}}\right)+\frac{1}{6}\left(\frac{P_{0}\left|\alpha_{0,0}\right|^{2}}{\mu_{\psi}+\sqrt{3} \sigma_{\psi}+\frac{N_{0}}{2 T_{b}}}\right) \\
& +\frac{1}{6}\left(\frac{P_{0}\left|\alpha_{0,0}\right|^{2}}{\mu_{\psi}-\sqrt{3} \sigma_{\psi}+\frac{N_{0}}{2 T_{b}}}\right) .
\end{aligned}
$$

\section{Simulation Results}

The proposed model has been simulated for indoor (line-ofsight), free space, urban, and shadowed urban scenarios. We consider $\mathrm{M}$ users uniformly distributed at random locations throughout a circular cell. These users were located at distances in the interval $(1000,1200)$ meters from the base station (BS) for urban, shadowed urban, and free space scenarios, and at distances in the interval $(90,100)$ meters from the BS for indoor (LOS) scenario. For each user in the cell, channels are generated using the proposed model.

For the simulations in this paper we assume that the angle spread is $13^{\circ}$ (for urban area, shadowed urban and free space [1]), which corresponds to $a=0.0033$, and $R=1.5 \mathrm{~km}$. For indoor scenario, the angle spread is assumed to be $24.5^{\circ}$, which corresponds to $a=0.0 .028$, and $R=200 \mathrm{~m}$. In all simulations, we set the chip rate to $8 \mathrm{Mb} / \mathrm{s}$, the process gain $N$ to 32 , and the number of multipath components $L_{k}=20$. The receiver is assumed to be omnidirectional. Finally, the SEIGA for the BER and SIR in (24) and (25) respectively are used to analytically determine the BER and SIR for each user.

Fig. 5 and 6 show the BER and SIR performance as a function of the number of users under different propagation scenarios. The propagation scenarios are: 1) urban area with a path loss exponent of $n=3,2$ ) shadowed urban with a path loss exponent of $n=4,3$ ) free space with a path loss exponent of $\mathrm{n}=2$, and 4) indoor (LOS) scenario with a path loss exponent of $\mathrm{n}=1.6$. The number of multipath components is set to $L_{k}$ $=20$. As illustrated in Fig. 5, the system is able to support 10 users in a free space and indoor scenarios at an average BER of $10^{-5}$ while the system is able to support 3 and 4 users for shadowed urban and urban scenarios, respectively.

\section{CONCLUSION}

A statistical geometric propagation model for a line-ofsight microcell mobile environment is proposed. This model 


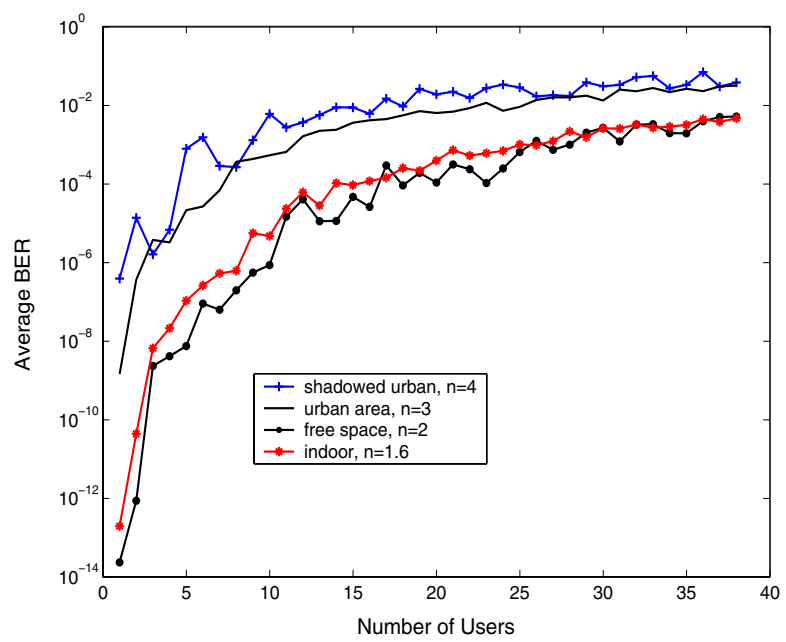

Fig. 5. BER performance as a function of the number of users under urban, shadowed urban, free space, and indoor propagation scenarios with $L_{k}=20$, the process gain $N=32$, and the chip rate $=8 \mathrm{Mb} / \mathrm{s}$.

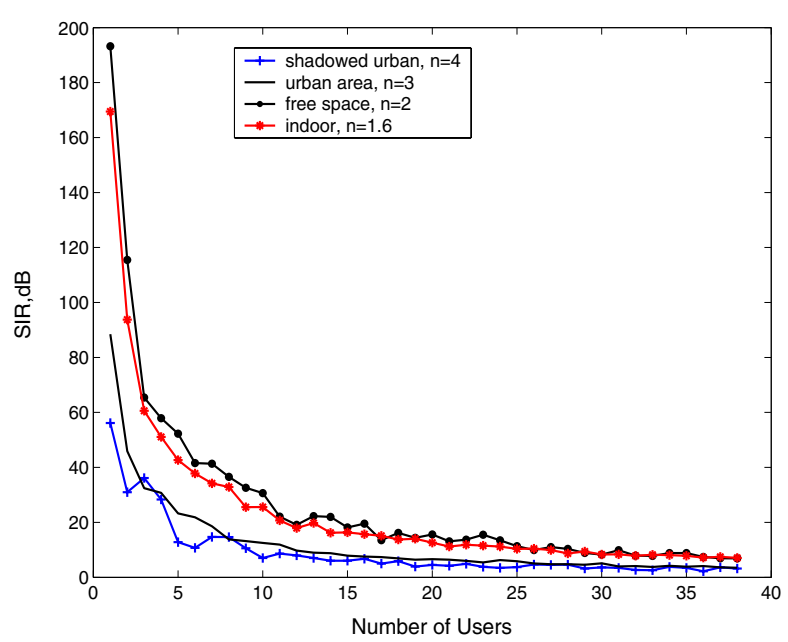

Fig. 6. SIR performance as a function of the number of users under urban, shadowed urban, free space, and indoor propagation scenarios with $L_{k}=20$, the process gain $N=32$, and the chip rate $=8 \mathrm{Mb} / \mathrm{s}$.

provides the power of each path, the TOA, and the DOA of the multipath component. The joint pdf of the powerDOA and the power-Doppler frequency shift for the proposed channel model are derived and simulated. The SEIGA in case of imperfect power control for wideband channel model has been used to evaluate the BER and the SIR. As expected, the DS-CDMA cellular system performs better in indoor and free space as compared with other scenarios (urban and shadowed urban). The pdf of the DOA for the proposed model has been numerically evaluated and compared with measurement data, where it appeared that the proposed model has a good match with the experimental results.

\section{ACKNOWLEDGEMENT}

The authors would like to thank Prof. R. Janaswamy from the Department of Electrical and Computer Engineering, Naval Postgraduate School, Monterey, CA, USA, and Dr. Q. H. Spencer, from the Distribution Control Systems, Inc., Hazelwood, Missouri, USA for their help in providing the practical measurement data that is used to validate the model.

\section{REFERENCES}

[1] J. C. Liberti and T. S. Rappaport, "A Geometrically Based Model for Line-of-Sight Multipath Radio Channels," Proc. of IEEE Veh. Tech. Conf, pp. 844-848, Apr. 1996

[2] Seedahmed S. Mahmoud, Zahir M. Hussain, and Peter O'Shea, “Spacetime model for mobile radio channel with hyperbolically distributed scatterers," IEEE Antennas and Wireless Propagation Letters, vol. 1, no. 12, pp. 211-214, 2002.

[3] _ "Geometrical model for mobile radio channel with hyperbolically distributed scatterers," The 8th IEEE International Conference on Communications Systems, vol. 1, pp. 17-20, Singapore, Nov. 2002.

[4] P. Petrus, J. H. Reed, and T. S. Rappaport, "Geometrical-based statistical macrocell channel model for mobile environments," IEEE Transactions on Communications, vol. 50, pp. 495-502, March 2002.

[5] Q. H. Spencer, B. D. Jeffs, M. A. Jensen, and A. L. Swindlehurst, "Modeling the Statistical Time and Angle of Arrival Characteristics of an Indoor Multipath Channel," IEEE Journal on Selected Areas in Communications, vol. 18, NO. 3, pp. 347-360, Mar. 2000.

[6] R. Janaswamy, "Angle and Time of Arrival Statistics for the Gaussian Scatter Density Model," IEEE Transactions on Wireless Communications, vol. 1, No. 3, pp. 488-497, July 2002.

[7] J. M. Holtzman, "A simple, accurate method to calculate spreadspectrum multiple-access error probabilities," IEEE Transactions on Communications, vol. 40, no. 3, pp. 461-464, March 1992.

[8] J. C. Liberti and T. S. Rappaport, "Accurate techniques to evaluate CDMA bit error rates in multipath channels with imperfect power control," Globecom'95, Singapore, pp. 33-37, Nov. 1995. 